\title{
BITrum: hacia un concepto interdisciplinar de la información
}

BITrum: towards an interdisciplinary concept of information

\author{
José María Díaz Nafría (1), Leticia BarRionuevo Almuzara (2)
}

(1) Universidad de León, Facultad de Educación, Campus de Vegazana, s/n 24071, jdian@unileon.es (2) Biblioteca Universitaria de León, Campus de Vegazana, s/n 24071 León, buffl@unileon.es

\begin{abstract}
Resumen
Se presenta el proyecto BITrum como un marco de investigación para una comprensión interdisciplinar de la información en todos sus aspectos, procurando abarcar el máximo de puntos de vista. Fundado en 2008 en la actualidad cuenta con más de 60 miembros de una decena de nacionalidades y disciplinas diversas que van desde las telecomunicaciones a la filosofía, desde la biología a la documentación, desde la física a las ciencias sociales. Se describen también las herramientas en red desarrolladas por BITrum para la constitución de una comunidad virtual de investigación para la compartición de recursos, facilitar la comunicación y el trabajo colaborativo. Finalmente se expone la iniciativa de internacionalización del proyecto a través de (1) la interconexión horizontal de toda la comunidad global de estudios informacionales (domusBITae) y (2) la propuesta de un programa de investigación transdisciplinar dentro del Espacio Europeo de la Ciencia.
\end{abstract}

Palabras clave: Ciencia de la información. Comunidad virtual de investigación. Concepto de información. Estudios informacionales. Interdisciplinariedad, Proyecto BITrum. Unificación. DomusBITae.

\section{Trasfondo, antecedentes e historia del proyecto}

En contraste con la incapacidad de aprehensión del concepto de hierro por parte de quienes habitaban en la llamada "edad de hierro", nosotros en la "edad de la información" sí podemos preguntarnos por la naturaleza de eso que llamamos información y a su vez proponer nuevas formas de comprender su contenido y su medida según se estudia y aplica en los más variados contextos científicos, técnicos y prácticos.

Se dice que hay información en células, palabras, antenas, cables, pensamientos, electrones, cerebros, robots, comunidades, bases de datos, instituciones [...] De modo que hablar de información es algo esencialmente transdisciplinar. Pero es más, por una parte, el concepto ha ganado un papel central en muchas disciplinas dispersando su significado y estableciendo brechas entre ellos (Capurro, 2003) y por otra par-

\begin{abstract}
The BITrum Project is launched as an interdisciplinary endeavour to understand information in all its variety, striving for gathering the most relevant points of view. Founded in 2008, it is and nowadays constituted by more than 60 members from different nationalities around the world covering knowledge fields from telecommunications to philosophy, from biology to library science, from physics to social sciences. Since its constitution, BITrum has developed a set of networking tools -here described- for the constitution of a virtual research community aimed at sharing resources, improving communication and enabling collaborative work. Finally, the initiative for the internationalisation of this virtual research community pursuing the horizontal interconnection of the global community of information studies- is exposed, followed by a scientific research programme planned for an effective interweaving of different frameworks to be deployed within the European Research Area.
\end{abstract}

Keywords: BITrum Proyect. Information. Information Science. Information studies. Interdisciplinarity. Unification. Virtual research community.

te, una aproximación teórico-informacional podría establecer nexos de unión entre disciplinas aparentemente irreconciliables, aportando soluciones a algunos enigmas científicos (Lyre, 2002). Es decir, a la vez que parece conducir a la incomunicación entre disciplinas científicas, se presenta también como promesa para acabar con su atávica distancia. Por otra parte, el mencionado efecto dispersivo en los significados de la información ha conducido a la creencia de que la información puede ser útil para cualquier cosa, mientras que frecuentemente no es suficiente para afrontar los problemas que pretenden resolverse con su concurso (Brier, 2008).

Puesto que estamos apelando a los entresijos de diversas ciencias, una clarificación de los fenómenos informacionales que provea a la vez rendimientos científicos y sociales debe ser, en consecuencia, interdisciplinar (Janich, 2008), siendo los principales problemas que habrían de abordarse -si se busca el máximo de compren- 
sión mutua sin reducir la diversidad fenoménica a la que atiende cada punto de vista-: (1) ¿Hay un grupo de conceptos bajo todos estos usos de "información"? (2) ¿Cómo puede garantizarse a la vez la diversidad de los fenómenos informacionales y el rigor de su aprehensión teórica? (3) ¿Puede un concepto refinado de información establecer un nexo de unión entre todos ellos? (4) ¿Cómo pueden preservarse todos los intereses prácticos respecto a la información?

\subsection{Antecedentes y orígenes}

El estado del arte en lo que atañe a las teorías de la información incluye muy diversos paradigmas en curso: complejidad (Teoría Algorítmica de la Información, info-computacional); entropía vs. orden (física, química); infónes (teoría de situaciones); sistema (cibernética); evolución (biología, teoría de sistemas), etc. Haciendo que disciplinas diferentes presupongan a menudo paradigmas distintos y de difícil reconciliación (Díaz y Salto, 2009).

Desde principios de los años de 1990 se han realizado esfuerzos significativos con objeto de erigir un marco interdisciplinar sobre la información. Entre ellos caben destacarse las acciones realizadas por: Foundations of Information Science (FIS), el Center for the Study of Language and Information (CSLI) de Stanford University, el Unified Theory of Information (UTI), el Institute for Logic, Language and Computation (ILLC) de Ámsterdam, y el Science of Information Institute (Soll) de Washington. Otros grupos como el International Center for Information Ethics (ICIE) o el Center for Information Policy Research (CIPR) fueron creados para velar por aquellos intereses relativos a la información pero que un tratamiento exclusivamente científico y tecnológico pudiera dejar de lado. Por otra parte, desde paradigmas o enfoques restrictivos se han realizado también notables esfuerzos por hacer una aproximación interdisciplinar a la información, como es el caso de la cibernética o la complejidad computacional.

BITrum constituye un intento interdisciplinar en el sentido antes propuesto (procurando abarcar el máximo de puntos de vista sin reducción fenoménica) que desde 2008 ha contado con la colaboración de alguno de los grupos internacionales antes mencionados (FIS, UTI, Soll, ICIE, CIPR) para desarrollar su programa.

\subsection{Proyecto y grupo de investigación BITrum}

"BITrum" se concibe como una metáfora que reúne el intentar dar cuenta de la información con el ensamblaje que caracteriza una vidriera (vitrum) en la que se convocan dentro de un mismo marco una multiplicidad de colores y formas que potencialmente se encuentran en la luz solar.

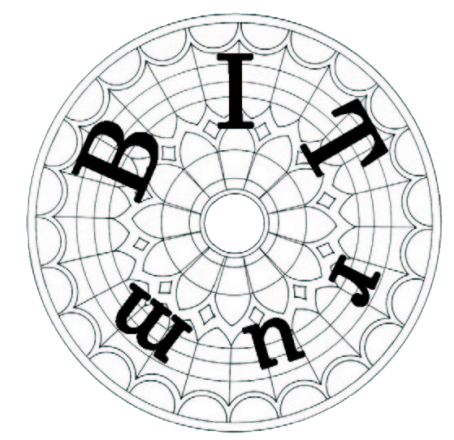

Figura 1. Logotipo del proyecto

El proyecto BITrum y su grupo de investigación, fundado en noviembre de 2008 con la ocasión del I Encuentro Internacional de expertos en teoría de la información - Un enfoque interdisciplinar, celebrado en León y que en la actualidad cuenta con más de 60 miembros de una decena de nacionalidades y disciplinas diversas que van desde las telecomunicaciones a la filosofía, desde la biología a la documentación, desde la física a las ciencias sociales [...] pretende encontrar un lugar común en el que los diversos puntos de vista respecto a la información se den cita, se aproximen en torno a problemas comunes y de interés general, con el objetivo de encontrar -desde el máximo rigor científico- mejores soluciones, nuevas maneras de indagación científica, de desarrollo tecnológico y de resolución de problemas sociales.

De forma sintética el objetivo para el proyecto y el grupo de investigación consiste en "elucidar las nociones de información de modo interdisciplinar, buscando definir nociones máximamente generales sin desatender los intereses y objetivos que alberga cada punto de vista y distinguiendo diferentes ámbitos de análisis: conceptos, metáforas, teorías, consecuencias y aplicaciones"; buscando así mismo que las nociones clarificadas sean funcionales y científicamente fructíferas (BITrum, 2008).

Dicho objetivo sintético se articula a su vez en 1) desarrollar un programa de investigación interdisciplinar para aclarar y articular los diferentes conceptos científicos de información y su relación con problemas teóricos y sociales; 2) constituir un grupo de referencia nacional en el campo de teoría de la información y promover en dicho campo la discusión científica en castellano; 3) abrir espacios de investigación internacional para la integración de las diferentes tenta- 
tivas internacionales de fundación de una ciencia de la información (con un enfoque interdisciplinar $y$, por lo tanto, marcadamente postpositivista).

La combinación de (2) y (3) se sustancia en el hecho de que los contenidos, las contribuciones científicas y las discusiones temáticas se reflejan tanto en castellano como en inglés, siendo bilingües las herramientas virtuales de participación a las que luego nos referiremos.

\subsection{Metodología de trabajo interdisciplinar}

La articulación de la investigación interdisciplinar responde al modelo sintetizado en la figura 1 (ver apéndice), donde caben distinguirse cuatro fases en la elaboración del proyecto y tres ámbitos en los que dicho trabajo tendría lugar. Siendo las fases para la articulación diacrónica: (1) la determinación de objetivos concretos (mediante una primera aproximación de los puntos de vista participantes); (2) planificación (distribuido en grupos de trabajo con objetivos concretos y consensuados); (3) desarrollo (principalmente dentro de los grupos de trabajo, pero con la posibilidad de un acceso a los distintos grupos); (4) Integración, evaluación y diseminación de los resultados.

Siendo los ámbitos de desarrollo sincrónico: (a) disciplinar o de grupos de trabajo concreto (orientados de acuerdo a unos problemas particulares y un referente teórico); (b) coordinación (con objeto de garantizar el cumplimiento de los hitos programados); (c) interdisciplinar (abiertos a la contribución de todos los participantes).

Como puede observarse en la figura 1 se distinguen así mismo, desde un punto de vista estratégico, las fases de constitución, consolidación del grupo nacional e internacionalización de la investigación. Para el desarrollo de estas fases el proyecto se apoya tanto en instituciones nacionales como internacionales de respaldo a la investigación así como en la ya mencionada colaboración con científicos destacados y grupos de investigación internacionales dentro del área.

\section{Portal BITrum como herramienta para la vertebración de la comunidad virtual de investigación}

Dada la dispersión geográfica de todos los integrantes que trabajan en el proyecto, resulta fundamental la existencia de un portal web que vertebre e integre todas las herramientas necesarias para el trabajo colaborativo y que sirva como medio de comunicación y difusión de información de todos los componentes de la co- munidad virtual de investigación. El portal BITrum desarrollado principalmente con las aplicaciones gratuitas de Google y Wordpress incluye cinco áreas que a continuación se detallan.

2.1. "Glossarium BITri: Glosario (bilingüe) de conceptos, metáforas, teorías y problemas en torno a la información"

Una de las primeras actividades iniciadas tras la constitución del Grupo BITrum en noviembre de 2008 y que ha servido de principal instrumento para el trabajo de clarificación interdisciplinar es el "Glossarium BITri" (figura A2, ver apéndice). Se trata de una herramienta fundamental para la articulación de la comunidad virtual ya que, por un lado, sirve como aproximación interdisciplinar a la noción de información, permitiendo el acceso a problemas desde diferentes perspectivas, y por otro, pretende reflejar el estado de la investigación dentro del marco del proyecto como fruto del trabajo de los grupos y de controversias entre puntos de vista diferentes. El glosario constituye así un pilar de la comprensión mutua entre los puntos de vista participantes.

Para favorecer la gestación multi-perspectivista, a la vez que colaborativa de los artículos que conforman el glosario, cualquiera de los miembros de BITrum o invitados especiales pueden contribuir con entradas independientes a cada una de las voces abiertas en el glosario. Cada voz tiene asignado un editor que normalmente contribuye a la clarificación de la voz y que se compromete a reflejar las entradas relevantes realizadas por otros autores de un modo sistemático, sin redundancias y en versión bilingüe castellano-inglés. Finalmente los coordinadores junto con el equipo de corrección se encargan de la supervisión, cumplimiento de los objetivos de calidad, administración del trabajo y comunicación entre las partes.

El glosario dispone de otras herramientas de información, acceso a contenidos y coordinación editorial: (i) índices en castellano e inglés, cuadro de cambio recientes y una herramienta de búsqueda para facilitar la navegación; (ii) una introducción al alcance, objetivos y metodología de la obra; (iii) un manual de instrucciones respecto a la incorporación, modificación, corrección... de contenidos; (iv) un libro de administración para el registro del trabajo realizado, y pendiente; ( $v$ ) registro para la proposición de nuevas voces; (vi) apartado de novedades que incluye anuncios relevantes respecto al glosario. las noticias más relevantes.

En el área de publicación de contribuciones (§2.4) se reflejan los artículos que satisfacen los 
criterios de publicación. Finalmente, una selección de artículos en inglés es publicada en una sección especial de la revista científica internacional TripleC.

\section{2. Área Pública}

Constituye principal zona de difusión pública del proyecto vertebrada en cinco secciones (figura A3, ver apéndice): 1. Proyecto BITrum, objetivos y alcance del proyecto, 2 . integrantes y organización del grupo, 3. Actividades BITrum, foros de discusión y difusión, propuestas a programas de investigación pública, congresos y reuniones de científicas, iniciativas de investigación o desarrollo, etc. 4. Recursos útiles para el investigador o interesado en el concepto de información y sus teorías, 5. Anuncios relacionados con el proyecto y sus miembros (pasos destacables, reuniones, acuerdos, incorporaciones al grupo, logros...). Entre los recursos que se ofrecen al investigador cabe destacarse: (a) "Enlaces" a información y recursos de interés para el proyecto clasificados en cinco categorías: instituciones asociadas, instituciones relevantes en teorías de la información, publicaciones científicas, páginas personales de los miembros y vídeos/presentaciones relativos al concepto de información; (b) "Archivo" de publicaciones editadas dentro del marco del proyecto; y (c) "Convocatorias" a congresos, artículos o proyectos dentro del marco de interés de BITrum.

\section{3. Área Privada}

Este espacio contiene información confidencial para uso de los miembros, como propuestas en desarrollo colaborativo, textos previos a su publicación, foros temáticos u organizativos, así como para la publicación de avisos de carácter interno, convocatorias o reuniones (figura 4, ver apéndice). Todos los miembros de BITrum tienen acceso a este área como lectores, y con capacidad de edición aquellos que participan en el trabajo de algunas de las actividades aquí reflejadas (incluidos los equipos de coordinación, de edición e informática). Entre los apartados a destacar dentro de éste área restringida, cabe resaltarse la Agenda donde se añadirán fechas clave, un directorio con datos personales de cada integrante del proyecto (nombre y apellidos, función dentro de BITrum, institución a la que pertenece, área del conocimiento, correo electrónico, teléfonos, así como un breve currículum vitae de cada uno) y el Archivo habilitado para compartir ficheros entre los componentes del proyecto.

\subsection{Bitácoras de Contribuciones y BITágora}

En el portal BITrum se han integrado dos bitácoras desarrolladas con WordPress destinados a la publicación de contribuciones científicas al proyecto y de discusión y debate temático orientado a todo el público interesado. Por un lado, la bitácora de Contribuciones (fig. 5 y 6 , apéndice) -con su doble faz bilingüe, aunque de contenidos no paralelos- cuyo objetivo es el de destacar las principales contribuciones al proyecto, ya sea en cuanto a artículos publicados en los encuentros que jalonan el desarrollo del proyecto, en revistas o congresos en los que se participe en calidad de desarrollo de la investigación de BITrum (e.g., la edición especial en TripleC de 2009) o contribuciones relevantes al glosario.

Como paso previo a una representación más elaborada de las relaciones semánticas dentro del campo de investigación de BITrum, la Nube BITrum -actualmente desplegada en las bitácoras- representa los términos clave que los propios autores consideran de mayor importancia en sus respectivas contribuciones. En ella los términos flotan de un modo independiente con un tamaño proporcional a la frecuencia de aparición en las contribuciones.

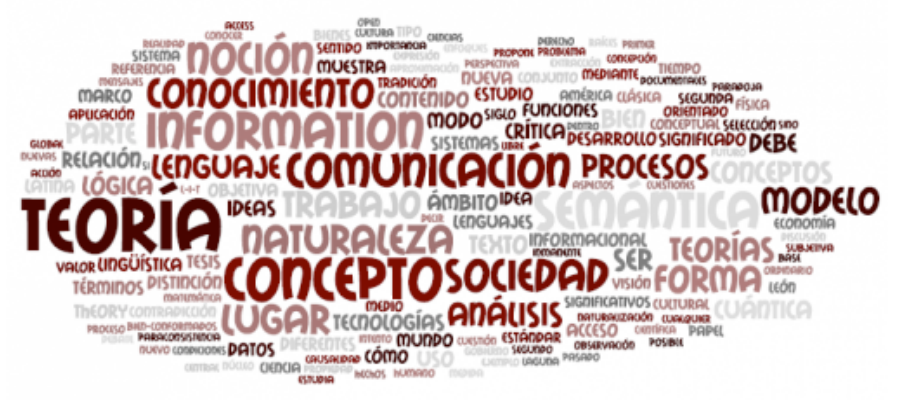

Figura 2. Nube bitrum

BlTagora (fig. 7), término formado por la conjunción de la unidad shannoniana de información con el espacio griego de discusión y acuerdo público -áyopá, desarrollado en colaboración con el Science of Information Institute, se concibe como un espacio para el debate sobre el concepto de información, de presentación y promoción del Science of Information Institute cuyo objetivo es la progresiva consolidación de una ciencia transdiciplinar de información. Contiene: entrevistas realizadas a científicos e investigadores relevantes que participan en este esfuerzo común; recensiones a libros relacionados con la materia y discusiones temáticas. 


\section{Perspectiva: internacionalización de la comunidad virtual de investigación}

Como parte de la estrategia de la comunidad virtual BITrum a la que antes nos referíamos (§1.3), su contextualización dentro de los problemas globales a los que pretende dirigirse implican la internacionalización tanto de (1) la comunidad virtual y de los recursos aportados al investigador como de (2) el programa científico que permita llevar a cabo la pretendida clarificación conceptual, teórica y práctica respecto a la información.

Para ello se plantea (1) la ampliación de la comunidad virtual de investigación hacia el conjunto de comunidades de estudios informacionales a nivel internacional especialmente europeo-, denominado "DomusBITae" y (2) el desarrollo de un programa científico que cabe interpretarse como la promoción de una nueva "ciencia de la información", bajo el apelativo de "bridging domains".

\subsection{Internacionalización de la comunidad científica: DomusBITae}

La iniciativa DomusBITae (metafóricamente formado por la encrucijada de las palabras latinas "Domus" y "Vitae" con la unidad de información propuesta por Shannon, bit) ha sido promovida a partir de 2009 por el grupo BITrum en colaboración con el Instituto Nacional de Tecnologías de la Comunicación (INTECO), el Science of Information Institute de Wahington (Soll), Foundations of Information Science (FIS), Unified Theory of Information Research Group de Austria (UIT), Universidad de León, Universitat de Barcelona, University of the Aegean, Grecia, Copenhagen Business School, Dinamarca, y Mälardalen University, Suecia.

Su objetivo es el de desarrollar una comunidad virtual de estudios informacionales (esto es, los dedicados al estudio de la información en alguno de sus aspectos), fomentando la constitución de una nueva ciencia de la información, como marco en el que tales estudios puedan efectivamente colaborar en la confrontación a los problemas sociales, técnicos y científicos que plantea la 'información'. Persigue una interconexión horizontal de toda la comunidad de estudios informacionales para: compartir recursos y resultados; mejorar la comunicación; fomentar la discusión, el conocimiento científico y la innovación; así como promover la investigación cooperativa.

El sistema propuesto -tal y como esquematiza la figura 3- contendría los siguientes módulos:
(1) Un sistema web orientado a una gestión efectiva del conocimiento para cualquier comunidad de estudios informacionales; (2) Un directorio de comunidades a modo de puente entre comunidades; (3) Un repositorio temático e institucional de estudios informacionales; (4) Herramientas para el trabajo en grupos para facilitar la investigación colaborativa; y (5) Un glosario compartido para la clarificación conceptual, la desambiguación teórica, y una aproximación multiperspectivista a los problemas informacionales.

La comunidad virtual DomusBITae se entiende como la confederación de comunidades específicas instanciadas por portales que utilizan un patrón común de acceso a las herramientas y recursos tanto privativos (de la comunidad específica) como confederados, mediante un sistema coordinado de identificación. El directorio sirve de nexo para moverse dentro de la comunidad virtual DomusBITae.

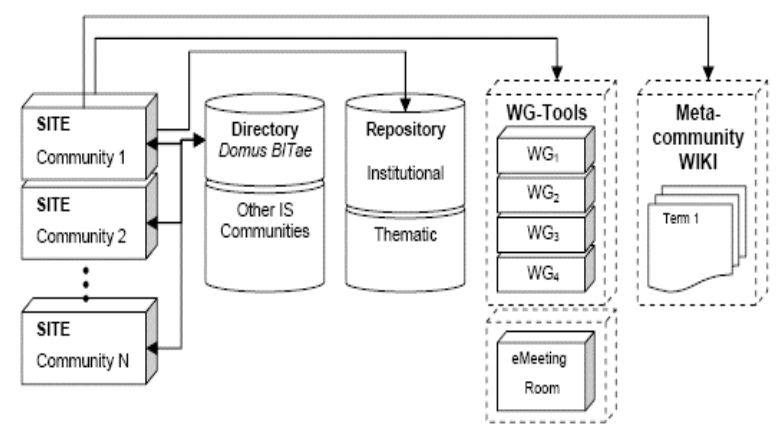

Figura 3. Estructura del sistema DomusBITae

Para alcanzar una máxima efectividad e impacto del sistema, el diseño se ha planificado con objeto de ser desarrollado por una representación relevante de la Comunidad Científica, observando el consentimiento de la comunidad y la adaptación a sus necesidades. Por otra parte un Comité Científico, representante de la comunidad investigadora y que ya reúne investigadores de gran relieve internacional (como R. Capurro, L. Floridi, W. Hofkirchner, S. Brier, M. Burgin, etc.), cumple la misión de garantizar un nexo entre el desarrollo técnico (despliegue de la infraestructura) y la usabilidad científica del mismo (programas científicos a los se encomienda y en particular el que a continuación se describe, §3.2).

Un consorcio constituido por las instituciones antes mencionadas y abierto a nuevas incorporaciones es el solicitante de financiación dentro de los marcos de apoyo a la actividad investigadora de la Comisión Europea en las líneas de infraestructuras electrónicas. 
Como núcleo del sistema para su promoción y puesta en marcha el sistema se está desarrollando para albergar de manera preliminar el portal de Fundations of Information Science (FIS) incluido su excepcional archivo de discusión conceptual en torno a la información, el portal del Science of Information Institute (Soll) y el Glossarium BITri.

\section{2. "Bridging domains": Una aproximación} multidimensional a la información

Con objeto de promocionar la internacionalización de la investigación conceptual en torno a la información la comunidad virtual BITrum en cooperación con otros investigadores y comunidades científicas de diversos países ha propuesto un programa de investigación para su desarrollo dentro de los programas europeos de soporte a la investigación (European Science Foundation y Programas de la Comisión Europea).

Dicha propuesta se basa en la interrelación de la actividad científica existente articulada en marcos de trabajo que permitan a la vez la profundización en los fenómenos informacionales y un tratamiento interdisciplinar de problemas teóricos, técnicos y prácticos relacionado con la información.

Para ello -y en consonancia con la metodología de BITrum pergeñada en la fig. 1- se prevé el desarrollo de la actividad en términos de tres niveles: (a) marcos de trabajo sobre objetivos interdisciplinares específicos; (b) colaboración entre marcos de trabajo; (c) global para la discusión y la representación del interés general; que diacrónicamente se articulan a tenor del desarrollo de los programas de investigación que cifren los Grupos de trabajo participantes.

Los marcos de trabajo se basan en grupos de interés preexistente dentro de la comunidad participante con posibilidades de ampliarse a individuos y comunidades científicas. Por otra parte, estos marcos se conciben como entidades porosas en los que los participantes pueden entrar o salir de ellos y participar en grupos de trabajo que simultáneamente contribuyan a varios.

Los resultados de la investigación pueden considerarse como fruto del trabajo colaborativo dentro y a través de dichos marcos, que son: (1) fundamentación formal y matemática de las teorías de la información máximamente general (de modo que queden incluidas todas las aproximaciones matemáticas y formales); (2) aproximación info-computacional desde la que la información se concibe como una estructura y la computación como su dinámica; (3) organización de la materia, desde la información cuántica a la complejidad de las moléculas; desde el universo holográfico a la indeterminación de la observación; (4) aproximación bio-informacional, desde la complejidad molecular a la vida y a la cognición; evolución, información genética y procesos neuronales; (5) aproximación comunicacional, en torno a la complejidad de los procesos de significado, comunicación e interpretación; (6) aproximación ético-social, en torno a cuestiones éticas, críticas y de sociedad de la información; (7) aproximación sistémica, la auto-organización desde las estructuras físicas a las sociedades complejas; (8) gestión de la información, desde los datos a una sociedad de la información sostenible; (9) filosofía de la información, que cabe entenderse como una articulación de preguntas relevantes, clarificación de conceptos y refinamiento de argumentos.

\section{Conclusiones}

El proyecto BITrum se erige como una iniciativa innovadora, colaborativa e interdisciplinar, y como tal, creemos que su futuro es muy prometedor. Dentro del curso del proyecto, caben destacarse los siguientes pasos: (1) se ha partido de un programa de investigación; (2) se ha conformado una comunidad virtual de investigación incipientemente internacionalizada y (3) se han desarrollado herramientas y recursos para la investigación, con productos ya consolidados y de impacto creciente. Por lo que cabe esperar que las propuestas planteadas, tanto para la comunidad virtual de investigación, como para el programa de investigación se hagan pronto realidad con el apoyo de instituciones internacionales.

\section{Referencias}

BITrum (2008). Objetivos (del proyecto BITrum). http://sites.google.com/site/proyectobitrum/proyecto/1-2objetivos (2010-04-10).

Brier, Søren (2008). Cybersemiotics: why information is not enough. Toronto: University of Toronto Press.

Capurro, Rafael (2003). The concept of Information. // Annual Review of Information Science and Technology. 37:8 (2003) 343-411.

Diaz Nafría, J.M.; Salto Alemany, F. (2009). Special issue: "What is really information? An interdisciplinary approach." // TripleC. 7:2.

Google Sites. [online] http://www.google.com/sites (2010-0410)

Janich, Peter (2008). Was ist Information? Kritik einer Legende ¿Qué es información? Crítica de una leyenda. Frankfurt: Suhrkamp.

Lyre, Holger (2002). Informationstheorie. Eine philosophischnaturwissenschaftliche Einführung. [Teoría de la infor- 
mación. Una introducción filosófica y naturalista]. Munich: Wilhelm Fink.
Recibido: 15-04-2010. Revisado: 07-07-2010.

Aceptado: 07-07-2010

\section{Apéndice}

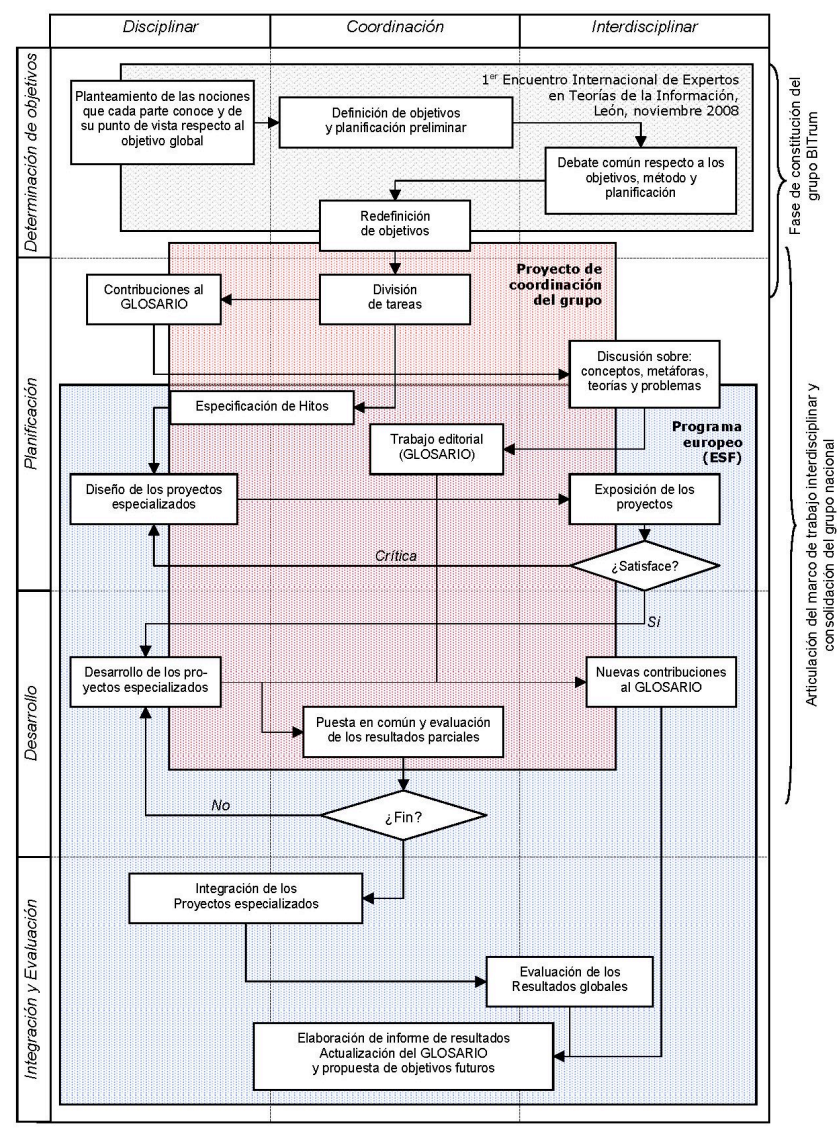

Figura 1. Metodología para el trabajo interdisciplinar (proyecto BITrum).

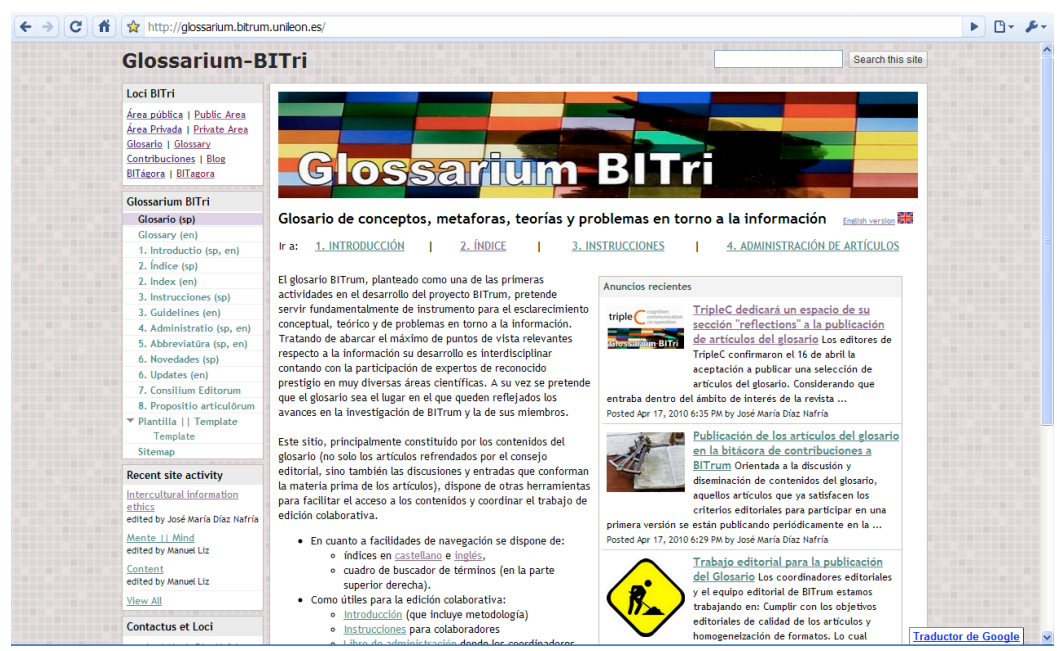

Figura 2. Glosario BITrum (pagina principal en castellano) 


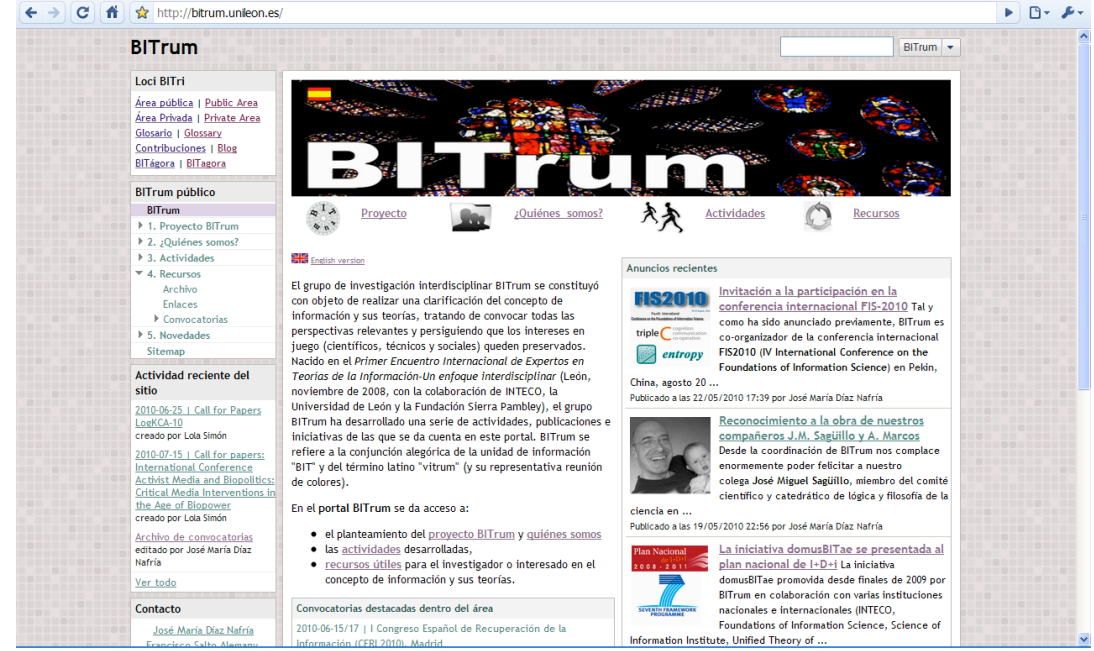

Figura 3: Área Pública en castellano (página principal)

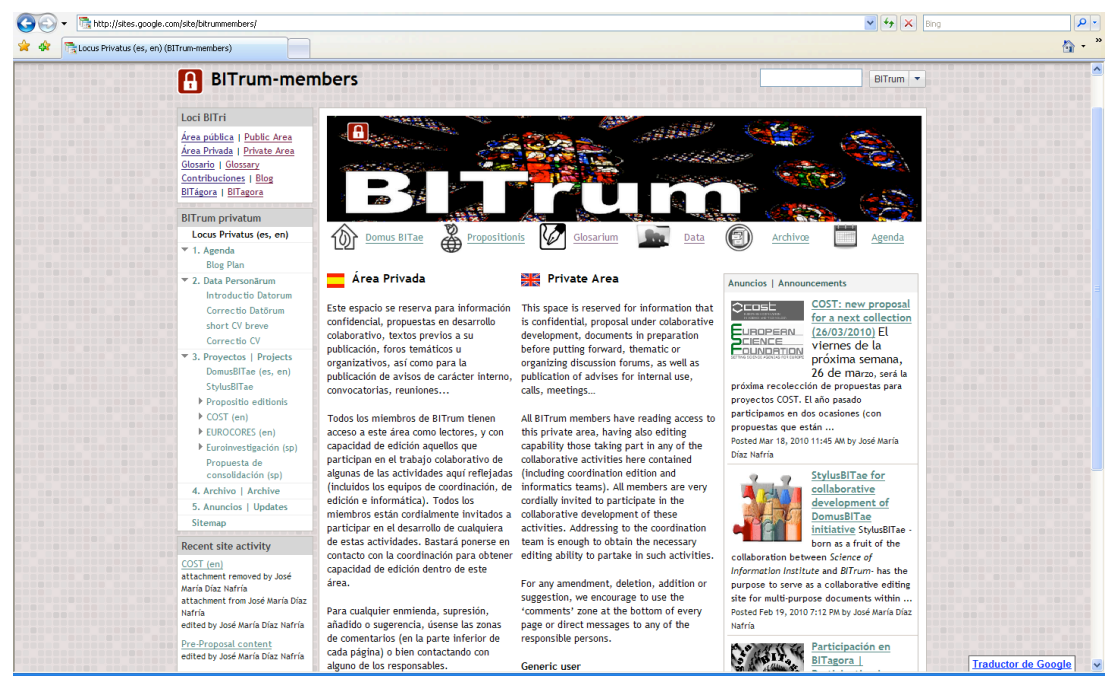

Figura 4. Área Privada (bilingüe)

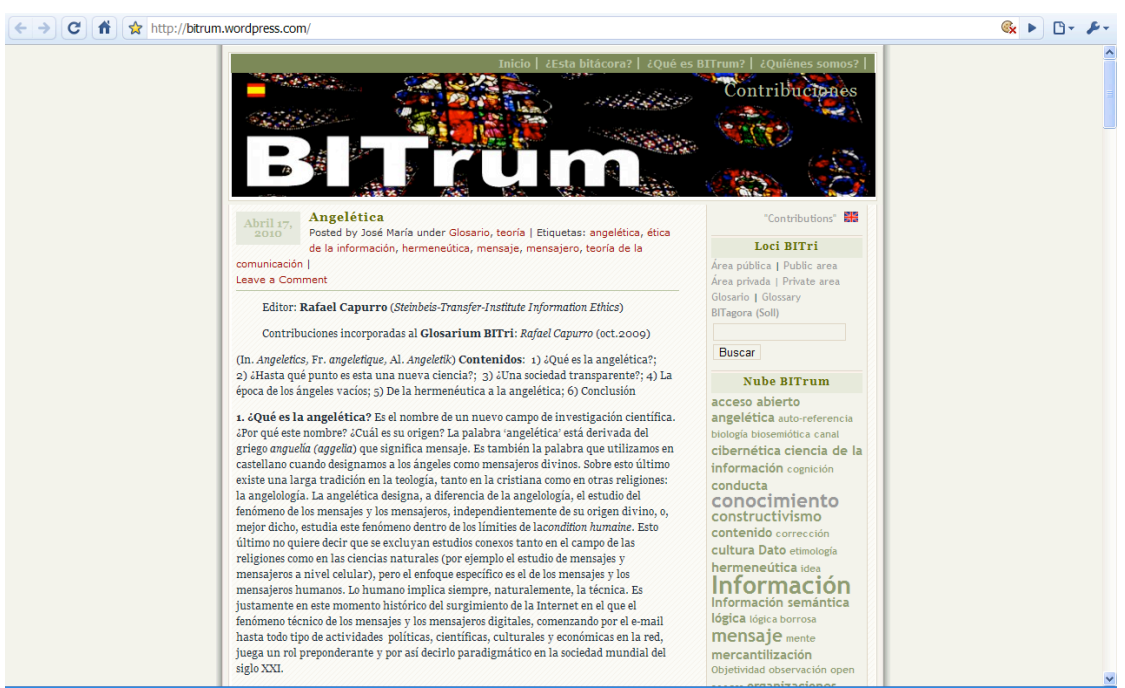

Figura 5. Bitácora de contribuciones en castellano 


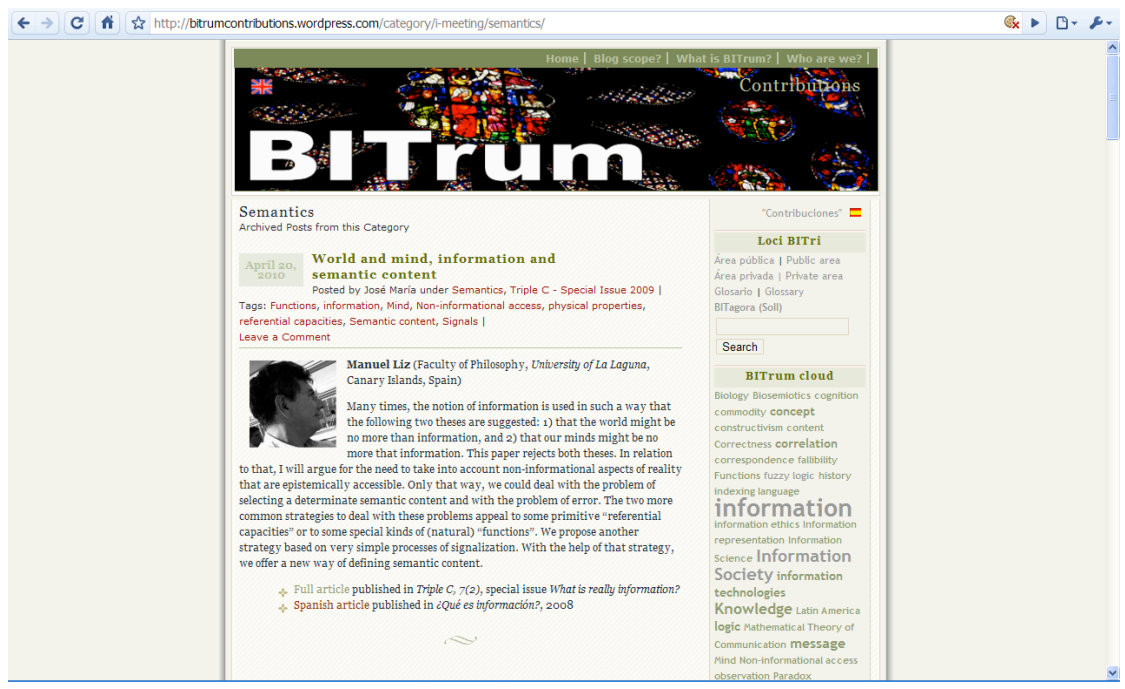

Figura 6. Bitácora de contribuciones en inglés

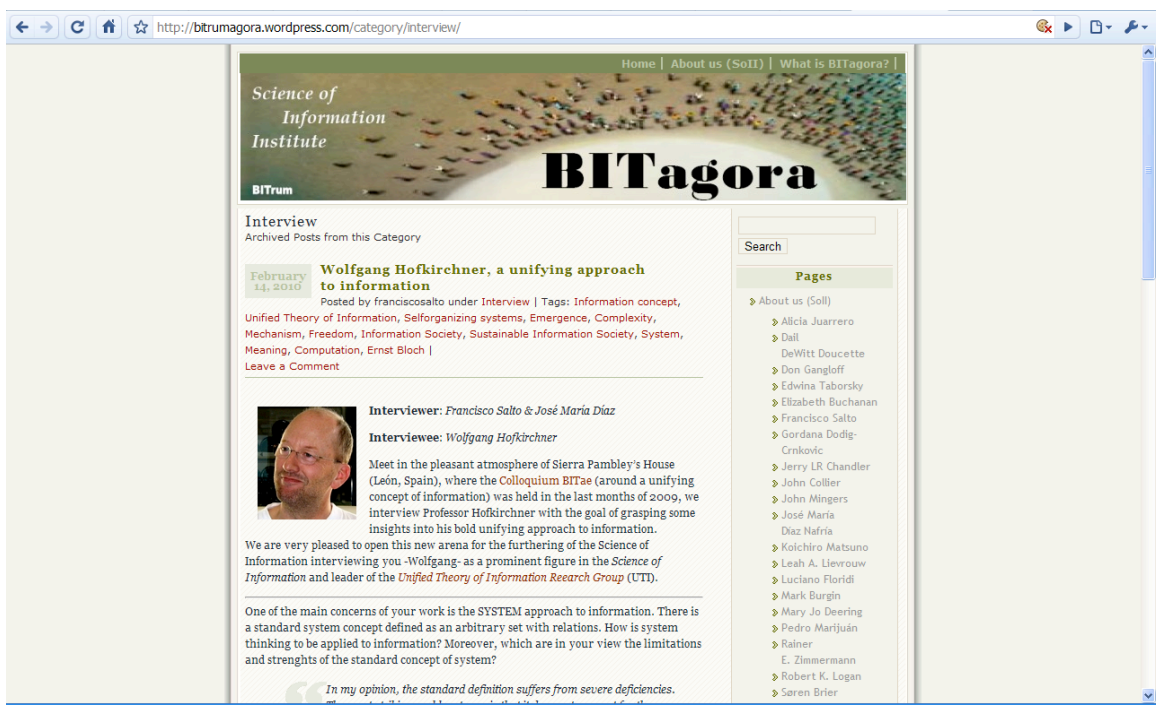

Figura A7. BITagora 
